\title{
Atmospheric pressure plasma deposition of antimicrobial coatings on non-woven textiles ${ }^{\star}$
}

\author{
Anton Yu. Nikiforov ${ }^{1}$, a, Xiaolong Deng ${ }^{1}$, Iuliia Onyshchenko ${ }^{1}$, Danijela Vujosevic ${ }^{2}$, Vineta Vuksanovic ${ }^{2}$, \\ Uros Cvelbar $^{3}$, Nathalie De Geyter ${ }^{1}$, Rino Morent ${ }^{1}$, and Christophe Leys ${ }^{1}$ \\ ${ }^{1}$ Department of Applied Physics, Ghent University, Sint-Pietersnieuwstraat 41, 9000 Ghent, Belgium \\ ${ }^{2}$ Center for Medical Microbiology, Institute of Public Health, DzonaDzeksona bb, 81000 Podgorica, Montenegro \\ 3 Jozef Stefan Institute, Jamova cesta 39, 1000 Ljubljana, Slovenia
}

Received: 27 October 2015 / Received in final form: 6 February 2016 / Accepted: 18 March 2016

(c) The Author(s) 2016

\begin{abstract}
A simple method for preparation of nanoparticle incorporated non-woven fabric with high antibacterial efficiency has been proposed based on atmospheric pressure plasma process. In this work direct current plasma jet stabilized by fast nitrogen flow was used as a plasma deposition source. Three different types of the nanoparticles (silver, copper and zinc oxide nanoparticles) were employed as antimicrobial agents. X-ray photoelectron spectroscopy (XPS) measurements have shown a positive chemical shift observed for $\mathrm{Ag} 3 d_{5 / 2}$ (at $368.1 \mathrm{eV}$ ) suggests that silver nanoparticles (AgNPs) are partly oxidized during the deposition. The surface chemistry and the antibacterial activity of the samples against Staphylococcus aureus and Escherichia coli were investigated and analyzed. It is shown that the samples loaded with nanoparticles of $\mathrm{Ag}$ and $\mathrm{Cu}$ and having the barrier layer of $10 \mathrm{~nm}$ characterized by almost $97 \%$ of bacterial reduction whereas the samples with $\mathrm{ZnO}$ nanoparticles provide $86 \%$ reduction of Staphylococcus aureus.
\end{abstract}

\section{Introduction}

Non-woven materials are used widely in applications ranging from medical dressing to everyday cleaning products due to its inherent advantages of large surface area, high porosity, absence of dust, good air permeability and ease processing [1]. In recent years, a lot of attention has been paid to achieve multifunctional performance of this fabrics, especially in health and hygiene field, health care and food packaging. Due to the potential for growth of pathogenic microorganisms which can lead and spread diseases, the non-woven fabrics with antimicrobial properties have gained significant attention $[2,3]$.

Usual procedure for obtaining antimicrobial textile material is textile finishing with antimicrobial agents. The traditional chemicals used nowadays in textile and biomaterials industry against broad spectra of bacteria face several disadvantages, including the worldwide emergency of antibiotic resistance, difficulties related to incorporation into many materials and sensitiveness to harsh environments during industrial processes and the wearing. Inorganic compounds of nanosize present strong antimicrobial activity at low concentrations due to their high surface

\footnotetext{
a e-mail: anton.nikiforov@ugent.be

* Contribution to the topical issue "6th Central European Symposium on Plasma Chemistry (CESPC-6)", edited by Nicolas Gherardi, Ester Marotta and Cristina Paradisi
}

area to volume ratio and unique chemical and physical properties [4]. Currently, incorporation of metal nanoparticles is becoming a new engineering pathway in the improving antimicrobial textiles activity because of the nanoparticles pronounced biocidal activity and superb stability under extreme conditions [5].

Several metal based nanoparticles have been extensively investigated as potential antimicrobial finishing agents. Among them, silver nanoparticles (AgNPs) have attracted most attention and merged up as a new generation of the antimicrobials with diverse medical applications [4]. On the other hand, zinc oxide nanoparticles (ZnONPs) is one of the five zinc compounds that are currently listed as generally recognized as safe (GRAS) by the U.S. Food and Drug Administration (21CFR182.8991) [6]. Many studies have shown that ZnONPs, have selective toxicity to bacteria and only exhibit minimal effect on human cells, which recommend their prospective uses in agricultural and food industries. Besides, great promise as antimicrobial agents have been reported for copper nanoparticles (CuNPs) against various microorganisms, and CuNPs expected to be a good candidate as an antibacterial agent for replacing $\mathrm{Ag}$ and other noble metal materials due to related environmental and economical benefits [7].

Recently, we proposed a novel method to prepare antimicrobial nanosilver polyethylene terephthalate (PET) non-woven fabrics by a three step process based on 


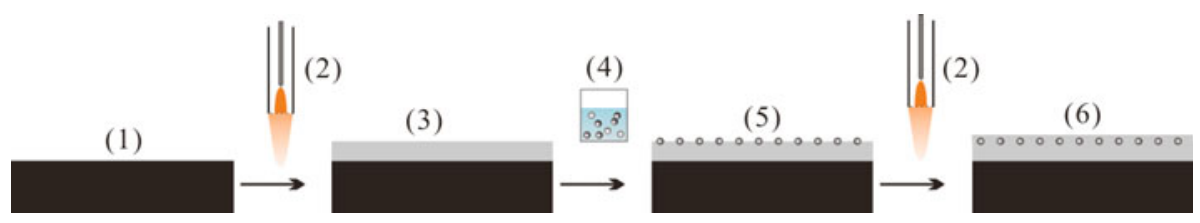

Fig. 1. Scheme of the fabrication process: (1) raw non-woven PET fabric; (2) plasma jet deposition system; (3) deposition of the 1st layer (reservation layer); (4) nanoparticle dispersion; (5) nanoparticle incorporation on the surface; (6) deposition of the 2nd layer (barrier layer). Reprint with permission from reference [8].

atmospheric pressure plasma deposition [8,9]. In this approach, AgNPs were firmly immobilized via a double layer of the plasma deposited organic films: a reservation layer (1st layer) and a barrier layer (2nd layer). It has been confirmed that the barrier layer could not only prevent the release of AgNPs, which is important to avoid potential toxicity in "real life" application, but can be also used to control the release of the silver ions, which is important in control of antimicrobial activity of the final products. It is important from practical point of view to explore functionality of the multilayer structures deposited by the plasma method with ZnONPs and CuNPs.

Herein, the work will be focused on study of incorporating feasibility of three different types of nanoparticles, i.e., AgNPs, ZnONPs and CuNPs, into the plasma deposited double layer structure. The surface chemistry of the materials is analyzed by XPS. The antimicrobial activity of the samples is tested against Staphylococcus aureus and Escherichia coli as the representatives of Gram-negative bacteria and Gram-positive bacteria, respectively. In addition, the attachment of nanoparticles to material and their fixation are validated by means of the washing tests carried out for non-woven PET with incorporation of AgNPs with and without the barrier layer.

\section{Experimental set-up and methods}

Nanoparticle non-woven polyethylene terephthalate (PET) fabrics are prepared using a three step procedure as shown in Figure 1. At first, an organosilicon thin film is deposited on the surface of the PET fabrics (DuPont, Spain) using the plasma deposited system, which is based on an atmospheric pressure DC plasma jet [10]. The raw PET fabric consists of fibres with an average diameter of approximately $10 \mu \mathrm{m}$ and a smooth surface. The direct current jet is operated at $20 \mathrm{~mA}$ current with about $50 \mathrm{~W}$ power dissipated directly in the discharge. All experiments are carried out with $\mathrm{N}_{2}$ as a main plasma gas with small admixing of $\mathrm{O}_{2}$ and hexamethyldisiloxane (HMDSO) which was used as precursor for the deposition of organosilicon coatings. The deposition is carried out only on one side of the non-woven PET as it was shown in our previous work [8], that uniform coating through the whole nonwoven fabric volume can be obtained in this way because of effective plasma penetration inside of the material. The total gas flow is set to $7 \mathrm{slm}$ (standard litres per minute) and a distance from top surface of the polymer to the plasma jet is fixed at $1 \mathrm{~cm}$. The first layer of $70 \mathrm{~nm}$ thickness is used as a reservation layer for the nanoparticles immobilization and a control of the nanoparticles adhesion to the PET fabrics. Then, the samples with plasma deposited layer are immersed into the suspension of the corresponding nanoparticles in ethanol and raised for drying. In the present work we use $20 \mathrm{~mL}$ of suspension prepared by sonification of the nanoparticles in pure ethanol for about $20 \mathrm{~min}$ following by immediate immersion of $1 \mathrm{~cm}^{2}$ of PET fabric in the solution for $1 \mathrm{~min}$. Three different types of nanoparticles, silver nanoparticles (SSNANO, USA) of $20 \mathrm{~nm}$ size, zinc oxide nanoparticles (SigmaAldrich, Belgium) of $50 \mathrm{~nm}$ size and copper nanoparticles (Sigma-Aldrich, Belgium) of $50 \mathrm{~nm}$ size are used in the experiments as purchased for the preparation of the corresponding nanocomposite fabrics. Four different concentrations of the nanoparticles in ethanol are used through the work of $1 \mathrm{mg} / \mathrm{mL}, 2 \mathrm{mg} / \mathrm{mL}, 5 \mathrm{mg} / \mathrm{mL}$ and $10 \mathrm{mg} / \mathrm{mL}$ with most of results (if not specially mentioned) given for the nanoparticles concentration of $5 \mathrm{mg} / \mathrm{mL}$. In the final step, a second layer of organosilicon film with a thickness of $10 \mathrm{~nm}$ is deposited. This layer is used as a barrier to prevent the release of the nanoparticles into the liquid medium [8]. The thickness of the deposited organosilicon film on non-woven PET cannot be measured directly due to complex 3D structure of the substrate. In order to determine the thickness an independent measurement with the deposition on PET film in identical experimental conditions has been carried out.

The surface chemistry of the films is determined by X-ray photoelectron spectroscopy (XPS) on a Versaprobe II system (Physical Electronics (PHI), USA) equipped with a monochromatic $\mathrm{Al} \mathrm{K} \alpha \mathrm{X}$-ray source $(h \nu=$ $1486.6 \mathrm{eV}$ ) and a pass energy of $23.3 \mathrm{~W}$. The pressure in the analyzing chamber is maintained below $10^{-7} \mathrm{~Pa}$ during analysis and the diameter of the analyzed area is $100 \mu \mathrm{m}$. The stoichiometry of the samples is calculated by comparison of the peak areas in survey spectra $(0-1100 \mathrm{eV})$, which are recorded at pass-energy of $117.4 \mathrm{eV}$, normalized by their corresponding sensitivity factor. All spectrums are background corrected for scattered electrons. The value of $285 \mathrm{eV}$ of the hydrocarbon $\mathrm{C} 1 s$ core level is used as a calibration of the energy scale.

Antimicrobial activity of the treated fabrics is tested by using macrodilution method against Escherichia coli (E. Coli, ATCC 25922) and Staphylococcus aureus (S. aureus, ATCC 25923), which are the most common pathogens, as the representative of Gram-negative bacteria and Gram positive bacteria, respectively. Before the 
assay, all samples are sterilized by a 30 min UV exposure. The samples of $0.5 \mathrm{~cm}^{2}$ are immersed in $3 \mathrm{~mL}$ of bacterial suspensions of culture $\left(10^{6} \mathrm{CFU} / \mathrm{mL}\right)$ and incubated for $24 \mathrm{~h}$ at $37^{\circ} \mathrm{C}$. Following incubation, the samples were transferred to $10 \mathrm{~mL} 0.9 \%(\mathrm{w} / \mathrm{v}) \mathrm{NaCl}$ and subjected to three cycles of $30 \mathrm{~s}$ vortex mixing and $30 \mathrm{~s}$ sonication. Tenfold serial dilutions were made in $0.9 \%(\mathrm{w} / \mathrm{v}) \mathrm{NaCl}$ and the number of CFU was determined by plate counting. Reduction of microorganisms $\mathrm{R}$ which indicates biostatic efficiency resulting from the contact with the samples is determined as:

$$
\mathrm{R}=(\mathrm{B}-\mathrm{A}) / \mathrm{B}
$$

where $\mathrm{A}$ and $\mathrm{B}$ is $\mathrm{CFU}$ per millilitre for the medium with the treated fabrics and the control samples after incubation, respectively. The sample with only first layer deposition is used as a control.

Mechanical stability/attachment of the coatings with double layer structure prepared by use of $5 \mathrm{mg} / \mathrm{mL}$ of AgNPs suspension is evaluated through the washing test of the samples after $1,3,5$ and 10 washing cycles for 40 min each in $200 \mathrm{~mL}$ of deionized water. All the samples were dried after the washing in a vacuum prior to the analysis. Due to time consuming analysis only samples of AgNPs were tested in the approximation that fixation of CuNPs and ZnONPs will show similar trends.

\section{Results and discussion}

The XPS survey spectra acquired from the control sample and the nanoparticle incorporated samples with $10 \mathrm{~nm}$ barrier layer are presented in Figure 2a. For the control sample corresponding to the fabric deposited with organosilicon film only, it was composited of Si, O and C. The appearance of $\mathrm{Ag}, \mathrm{Cu}$ and $\mathrm{Zn}$ peaks in XPS spectra for the samples after the nanoparticle incorporation reveals that the corresponding nanoparticles have been successfully decorated into the materials. An overview of the XPS measurements for the materials with incorporation of the nanoparticles is presented in Table 1.

We have found that the barrier layer presence of $10 \mathrm{~nm}$ reduces the XPS signal of $\mathrm{Ag}, \mathrm{Cu}$ and $\mathrm{ZnO}$ from initial (no barrier layer) values of $10.3,12.90$ and 21.64 at.\% to (10 nm barrier layer) 5.7, 6.9 and 8.6 at.\% respectively but still obvious signal of the incorporated metal nanoparticles is detected. The reason of fast drop in the intensity of XPS peaks for the samples with the barrier layer is related to the fact that XPS techniques has characteristic penetration depth of about $5 \mathrm{~nm}$ and thus the barrier layer will screen the presence of incorporated NPs. It is important to point out that the plasma deposition is carried out in pure nitrogen gas as the plasma forming gas of a flow $7 \mathrm{slm}$. On the other hand, there are no $\mathrm{N}$ atoms containing groups in the samples and no peaks of $\mathrm{N}$ atoms have been detected in the XPS measurements. This fact allows us to use $\mathrm{N}_{2}$ gas instead of much more expensive $\mathrm{Ar}$ or $\mathrm{He}$ and still have no incorporation of the undesirable $\mathrm{N}$ containing groups in the coatings.

Figures $2 \mathrm{~b}-2 \mathrm{~d}$ display the XPS high resolution spectra of silver, copper and zinc for those samples with the (a)
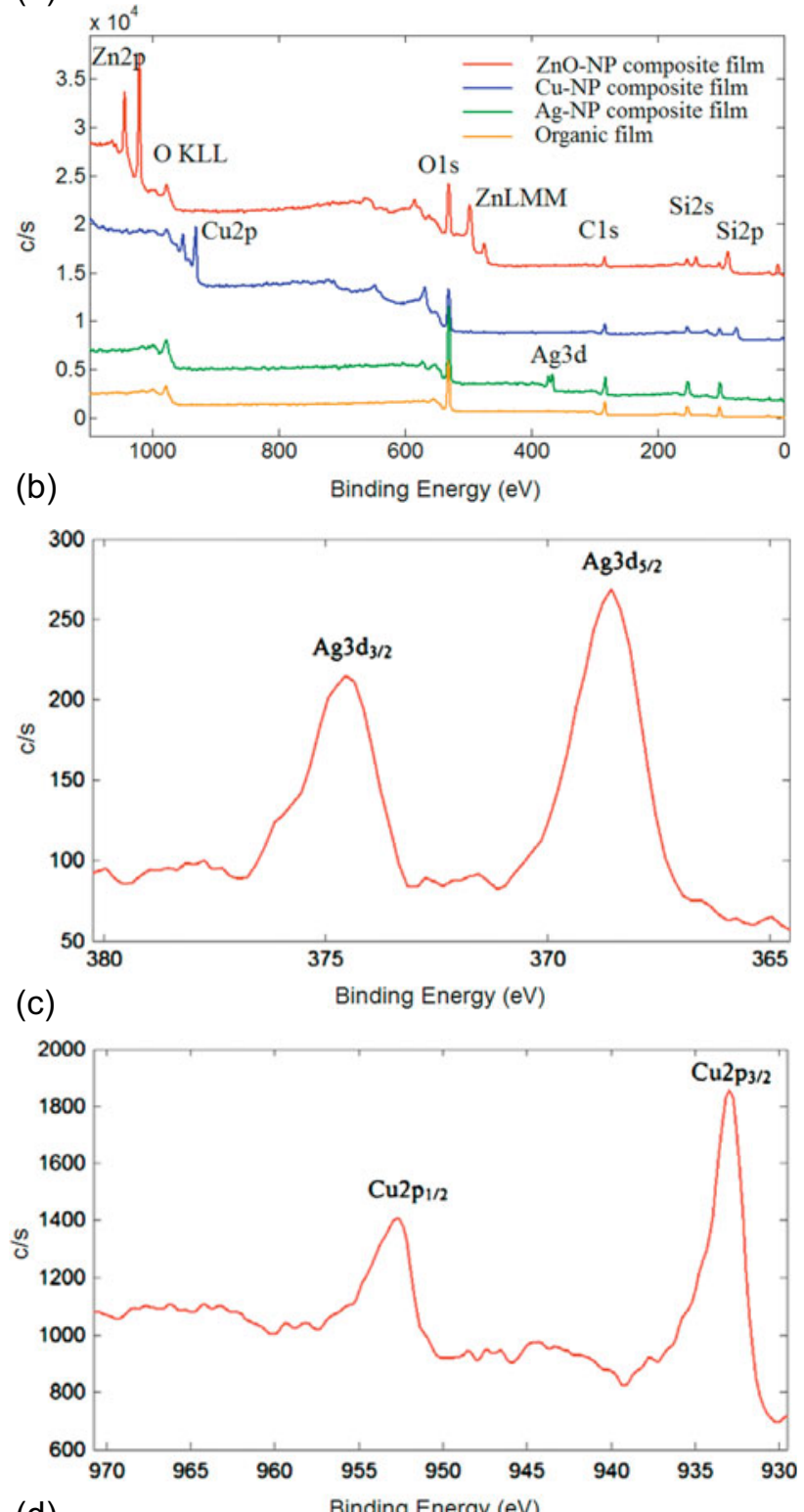

(d)

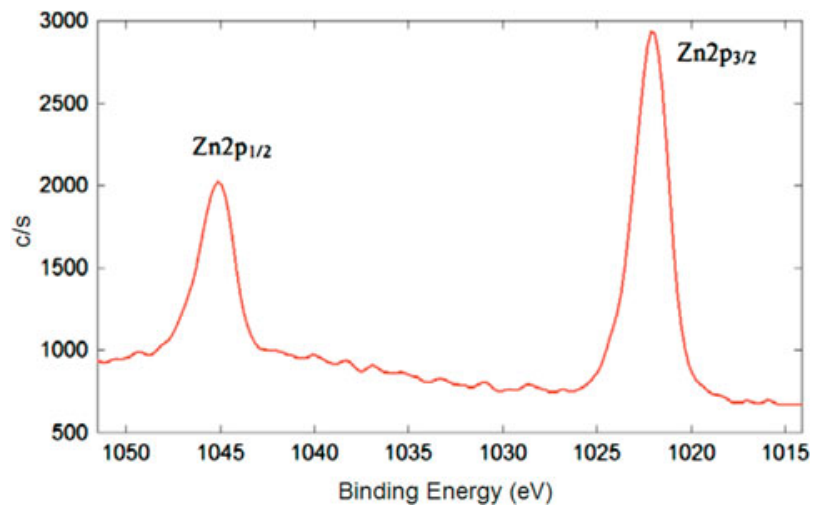

Fig. 2. XPS results of the nanocomposite films: (a) survey XPS spectra (presented spectra are shifted for convenience); (b) Ag $3 d$ XPS spectrum; (c) Cu $2 p$ spectrum; (d) Zn $2 p$ spectrum. Results presented on plots (b)-(d) correspond to materials with $10 \mathrm{~nm}$ barrier layer. 
The European Physical Journal Applied Physics

Table 1. XPS results of multi-layered coatings deposited on non-woven PET fabric. All results are averaged over 3 tested samples.

\begin{tabular}{ccccc}
\hline Sample & C $1 s$ & O $1 s$ & Si $2 s$ & $\mathrm{Ag} / \mathrm{Cu} / \mathrm{Zn}$ \\
\hline Reservation layer & $33.38 \pm 1.62$ & $45.85 \pm 1.12$ & $20.77 \pm 0.76$ & - \\
PET with AgNPs & $32.39 \pm 1.78$ & $42.67 \pm 1.16$ & $19.21 \pm 0.39$ & $5.73 \pm 0.37$ \\
PET with CuNPs & $28.07 \pm 0.34$ & $46.23 \pm 0.48$ & $18.77 \pm 1.03$ & $6.92 \pm 0.94$ \\
PET with ZnONPs & $23.99 \pm 1.02$ & $48.12 \pm 2.08$ & $19.25 \pm 0.86$ & $8.64 \pm 2.31$ \\
\hline
\end{tabular}

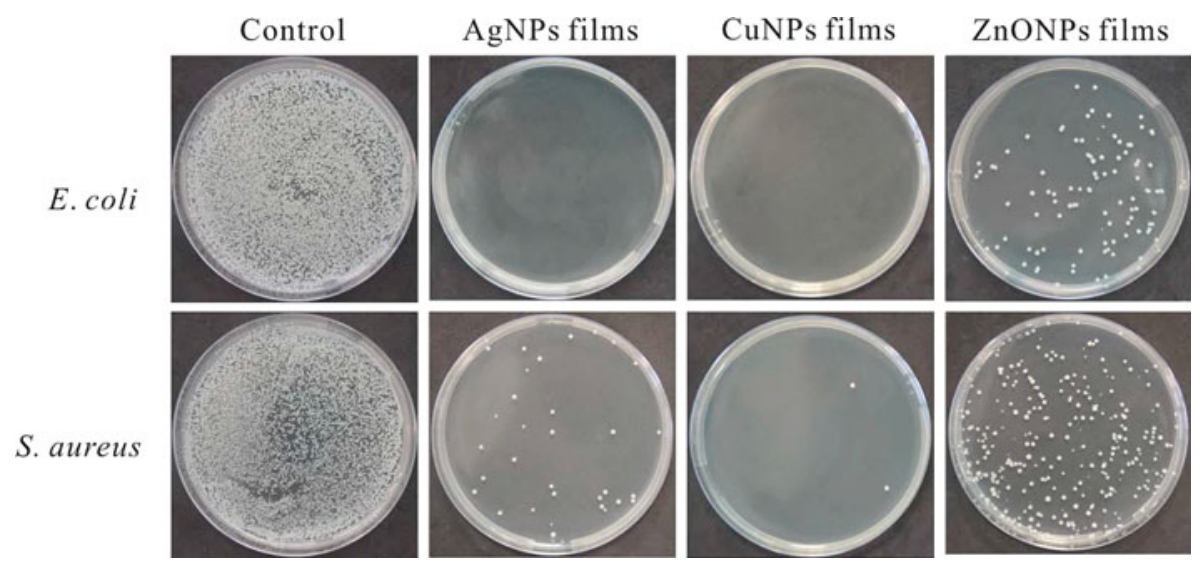

Fig. 3. Photographs of E. coli colonies and S. aureus on $\mathrm{MH}$ agar plates incubated with control samples and nanocomposite samples.

corresponding nanoparticles and $10 \mathrm{~nm}$ barrier layer. In Figure 2b, the peaks at $368.2 \mathrm{eV}$ and $374.2 \mathrm{eV}$ are assigned to $\mathrm{Ag} 3 d_{5 / 2}$ and $\mathrm{Ag} 3 d_{3 / 2}$, respectively. These peaks have a splitting of $3 d$ doublet with $6 \mathrm{eV}$ indicating the presence of metallic silver. While, comparing to the binding energy of $\mathrm{Ag} 3 d_{5 / 2}$ for bulk metal $\mathrm{Ag}$ at 368.2 $\mathrm{eV}$, a positive chemical shift observed for $\mathrm{Ag} 3 d_{5 / 2}$ (at $368.1 \mathrm{eV}$ ) suggests that silver nanoparticles are partly oxidized in the process [11]. The $\mathrm{Cu} 2 p_{1 / 2}$ and $\mathrm{Cu} 2 p_{3 / 2}$ (Fig. 2c) centered at $952.2 \mathrm{eV}$ and $932.4 \mathrm{eV}$ with a spinorbit separation of $18.8 \mathrm{eV}$ for the sample embedding $\mathrm{Cu}-\mathrm{NPs}$. The absent of shake-up peaks at about $941.5 \mathrm{eV}$ indicates no $\mathrm{Cu}^{2+}$ are presented in the sample [12]. However, it is difficult to identify $\mathrm{Cu}^{0}$ and $\mathrm{Cu}^{+}$due to the limitation of XPS resolution, as mentioned by Chen [13]. Figure 1d represents the XPS spectra of Zn $2 p$, and the peak position of $\mathrm{Zn} 2 p_{1 / 2}$ and $\mathrm{Zn} 2 p_{3 / 2}$ locate at $1045.2 \mathrm{eV}$ and $1022.2 \mathrm{eV}$, respectively.

The capability of the nanocomposite fabrics to prevent viable bacteria colonization was verified by the plate counting technique (PCT). Figure 3 shows typical colonization by $E$. coli and $S$. aureus on the Mueller Hinton $(\mathrm{MH})$ agar plates from the liquid medium cultured with the samples containing different types of AgNPs, similar results were obtained also for CuNPs and ZnONPs. Samples with barrier layer of $10 \mathrm{~nm}$ are used in antibacterial tests against of $E$. coli and $S$. aureus. Different concentrations of the nanoparticles of $1 \mathrm{mg} / \mathrm{mL}, 2 \mathrm{mg} / \mathrm{mL}$, $5 \mathrm{mg} / \mathrm{mL}$ and $10 \mathrm{mg} / \mathrm{mL}$, used during the dipping process, were tested. Compared to the fabrics without the nanoparticles, the PET fabrics treated by nanoparticle exhibited significant antibacterial activity against both bacteria, which clearly indicated that the growth of microorganisms in medium was affected by the presence of corresponding nanoparticles on the fabrics. It was found that the numbers of survived colonies of $E$. coli and $S$. aureus on the agar plates decreased for the samples with higher silver concentration. Almost complete reduction of $97 \%$ has been reached against $E$. coli at NPs concentration of $5 \mathrm{mg} / \mathrm{mL}$ for $\mathrm{Ag}$ and $\mathrm{Cu}$ but in $\mathrm{ZnO}$ reduction of $86 \%$ was observed at the same conditions. The bacterial reduction of $E$. coli and $S$. aureus for the samples with $10 \mathrm{~nm}$ barrier and $5 \mathrm{mg} / \mathrm{mL}$ nanoparticles load is presented in Figure 4.

As one can see from the figure, the samples incorporated three nanoparticles exhibits effective antibacterial activity against both microorganisms. In general, all nanocomposite PET fabrics were more effective against $E$. coli than $S$. aureus as also reported elsewhere [11]. Since the concentrations and the size of the incorporated nanoparticles are different, comparison on the antibacterial efficiency among the three types of nanoparticles requires additional investigation which is out of the scope of the present work.

It is important to note that all the materials produced by our method consist of double layers. The top layer is used to control release of the antimicrobial agents and also to prevent free desorption of the nanoparticles from the surface of the non-woven materials. In practical applications of the coatings the durability of the deposits in wearing/washing is a key point of the use. The durability of nanoparticles bonding in the plasma deposited matrix has been investigated through the washing process for AgNPs as a test object [8]. We found that the double layer structure has the ability to firmly immobilize 
A.Yu. Nikiforov et al.: Atmospheric pressure plasma deposition of antimicrobial coatings on non-woven textiles

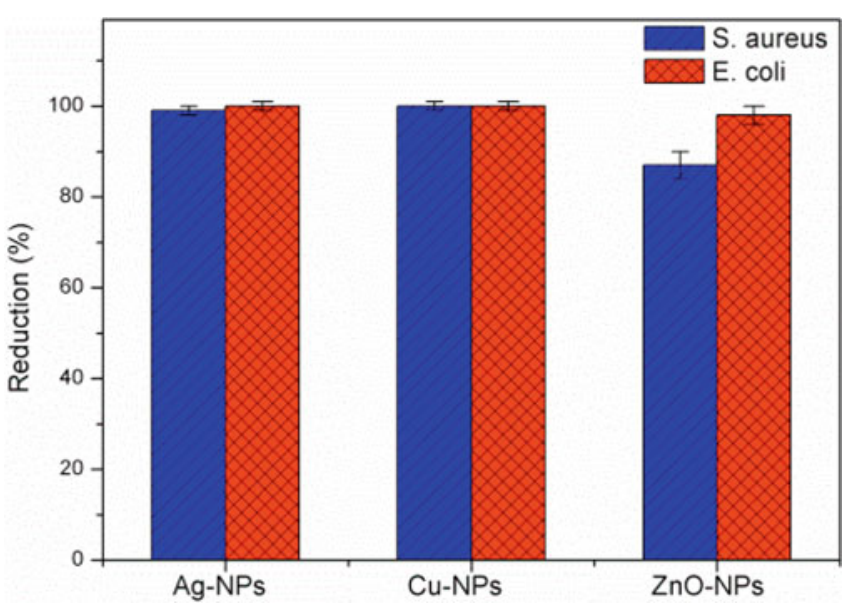

Fig. 4. Efficiency of the samples with three types of nanoparticles against E. coli and S. aureus. Each data point and error bar represents the mean and standard errors, respectively, of independent triplicates.

the incorporated nanoparticles during mechanical washing cycles, which suggests the release of the nanoparticles can be avoided in most of applications. For the samples containing $\mathrm{AgNPs}$, the silver concentration in the samples without the barrier layer showed a significant fluctuation after several washing cycles (see Fig. 5).

This is explained by desorption of physically absorbed AgNPs from the non-woven materials and migration to the medium during the washing process. For the samples with the barrier layer, however, effective immobilization of silver in the matrix was confirmed with stability of the antimicrobial effect even after 10 washing cycles. Thus, the direct interaction between the nanoparticles and the bacterial medium can be neglected. Therefore, we expect that the main antimicrobial mechanism is a release of active species from the surface of incorporated nanoparticles directly in liquid media. In work [14] it was shown that antibacterial activity of $\mathrm{ZnO}$ is attributed mostly to generation of $\mathrm{H}_{2} \mathrm{O}_{2}$ on the surface of the nanoparticles and furthermore reactivity of $\mathrm{H}_{2} \mathrm{O}_{2}$ in reactions with bacterium media. Similar mechanism of $\mathrm{ZnO}$ activity has been found in reference [15]. In reference [16] indeed it was confirmed that the release of $\mathrm{Zn}^{2+}$ ions from nanoparticles has negligible effect of on the growth of $E$. coli and $S$. aureus. In contrast for $\mathrm{Ag}$ and $\mathrm{Cu}$ nanoparticles the expected antibacterial affect is related to dynamics of corresponding ions release $[17,18]$. Lee [18] propsoed $\mathrm{Ag}^{+}$release in an acidic enviroment by the following reaction:

$$
2 \mathrm{Ag}(\mathrm{s})+1 / 2 \mathrm{O}_{2}(\mathrm{aq})+2 \mathrm{H}^{+} \rightarrow 2 \mathrm{Ag}^{+}(\mathrm{aq})+\mathrm{H}_{2} \mathrm{O}(\mathrm{aq})
$$

Similar process is expected to be responsible for the release of $\mathrm{Cu}^{+2}$ ions from CuNPs [19], however experimental works on study of CuNPs are scarce. The presence of nanoparticles in the coatings which are in direct contact with liquid would ensure continuous release of the ions into the nutrient media. $\mathrm{Ag}^{+} / \mathrm{Cu}^{2+}$ released by the nanoparticles may attach to the negatively charged bacterial cell wall and rupture it, thereby leading to protein

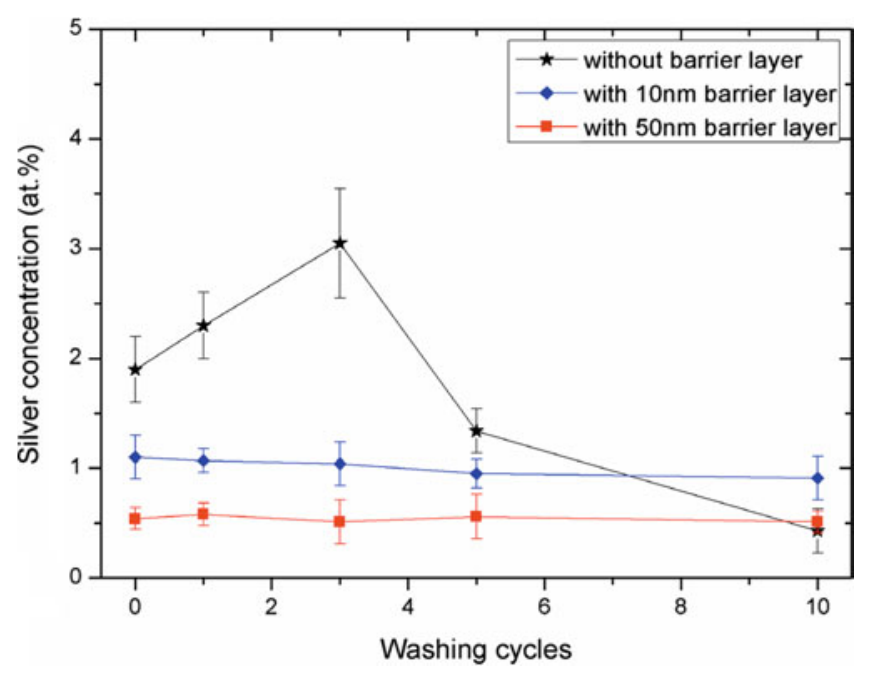

Fig. 5. Silver concentration on the PET samples without barrier layer, with $10 \mathrm{~nm}$ and $50 \mathrm{~nm}$ barrier layers after 0 , $1,3,5$ and 10 washing cycles. Reprint with permission from reference [8].

denaturation and cell death [20]. Number of different mechanisms responsible for interaction of metal ions with microorganisms structure has been proposed [21,22], e.g., oxygen associated with silver can react with the sulfhydryl $(-\mathrm{S}-\mathrm{H})$ groups on cell wall to form $\mathrm{R}-\mathrm{S}-\mathrm{S}-\mathrm{R}$ bonds thus, blocking respiration and causing death of cells. Lok et al. [23] have found that the surface of the cell walls of E. coli treated with AgNPs were severely damaged compared to untreated $E$. coli leading to cell wall rupture due to silver ions. Difference in the mechanisms of antibacterial efficiency in between $\mathrm{ZnO}$ and $\mathrm{Cu} / \mathrm{Ag}$ nanoparticles can explain observed here (see Figs. 3 and 4) different effect of them on bacteria but obviously more detailed investigation of active agent release from the composite coatings loaded with nanoparticles is required which is out of the scope of the present research.

\section{Conclusion}

Non-woven fabrics incorporated with three types of the nanoparticles, AgNP, CuNP and $\mathrm{ZnONP}$, have been prepared by a three step method based on atmospheric pressure plasma process. The XPS results reveal the nanoparticles have been successfully embedded into the fabrics. All nano fabrics show effective antimicrobial activity against $E$. coli and $S$. aureus. It is found that the use of CuNPs has almost the same efficiency as AgNPs whereas ZnONPs demonstrated lower efficiency against $S$. aureus. The results prove that the methods for the immobilization of nanoparticles in to the structure of non-woven fabrics might present a new route for preparation of highly effective antimicrobial materials for future application.

The work was supported by STSM Grant of the COST Action MP1101. The research leading to these results has also been partially supported by the European Research Council under 
the European Union's Seventh Framework Program (FP/20072013)/ERC Grant Agreement no. 279022 (PLASMAPOR) and ERC Grant Agreement no. 335929 (PLASMATS).

\section{References}

1. B.S. Liu et al., Macromol. Biosci. 8, 5 (2008)

2. G. Irena et al., Appl. Surf. Sci. 255, 19 (2009)

3. J.-P. Chen et al., Appl. Surf. Sci. 262, 95 (2012)

4. M. Rai et al., Biotechnol. Adv. 27, 1 (2009)

5. H. Palza, Int. J. Mol. Sci. 16, 1 (2015)

6. P.J.P. Espitia et al., Food Bioprocess Technol. 5, 5 (2012)

7. S. Chen et al., J. Mater. Chem. 22, 18 (2012)

8. X. Deng et al., Sci. Rep. 5, 10138 (2015)

9. X. Deng et al., Mater. Lett. 149 (2015)

10. X. Deng et al., Plasma Processes Polym. 10, 7 (2013)

11. X. Deng et al., Plasma Processes Polym. 11, 11 (2014)

12. Y.H. Kim et al., J. Phys. Chem. B 110, 49 (2006)
13. Z. Chen et al., Nanotechnology 24, 26 (2013)

14. J. Sawai et al., J. Ferment. Bioeng. 86, 521 (1998)

15. O. Yamamoto, Int. J. Inorg. Mater. 3, 7 (2001)

16. J. Sawai, J. Microbiol. Methods 54, 177 (2003)

17. N. Cioffi et al., Anal. Bioanal. Chem. 381, 3 (2005)

18. Y.-J. Lee et al., Environ. Toxicol. Chem. 31, 1 (2012)

19. N. Cioffi et al., Anal. Bioanal. Chem. 381, 607 (2005)

20. J.P. Ruparelia et al., Acta Biomater. 4, 707 (2008)

21. Y.E. Lin et al., Water Res. 32, 1997 (1998)

22. V. Siva Kumar et al., J. Mol. Catal. A Chem. 223, 313 (2004)

23. C. Lok et al., J. Proteome Res. 5, 916 (2006)

Open Access This article is distributed under the terms of the Creative Commons Attribution License http:// creativecommons.org/licenses/by/4.0 which permits unrestricted use, distribution, and reproduction in any medium, provided the original author(s) and source are credited. 